\title{
Rickettsia slovaca Infection: DEBONEL/TIBOLA
}

\author{
V. IBARRA, ${ }^{a}$ J.A. OTEO,$^{a}$ A. PORTILLO ${ }^{a}$ S. SANTIBÁÑEZ, ${ }^{a}$ \\ J.R. BLANCO ${ }^{a}$ L. METOLA, ${ }^{a}$ J.M. EIROS,${ }^{b}$ L. PÉREZ-MARTÍNEZ, ${ }^{a}$ \\ AND M. SANZ ${ }^{a}$ \\ a Área de Enfermedades Infecciosas, Hospitales San Millán-San Pedro-de La \\ Rioja, Logroño, Spain \\ ${ }^{b}$ Centro Nacional de Microbiología, Instituto de Salud Carlos III. Madrid, Spain
}

\begin{abstract}
This study describes the epidemiological, clinical, and microbiological characteristics of a new tick-borne disease in SpainDermacentor-borne necrosis erythema lymphadenopathy (DEBONEL). The clinical presentations include an eschar at the site of the tick bite, surrounded by an erythema and painful regional lymphadenopathy. The disease appears during the colder months and its vector is Dermacentor marginatus (D. marginatus). From January 1990 to December 2004, 54 patients presented at Hospital of La Rioja with these clinical and epidemiological data. The ratio of females to males was $32 / 22$. The average age was 37 years. In all cases tick bites were located on the upper body (90\% on the scalp). The median incubation period was 4.7 days. Signs and symptoms were mild in all cases. Only a small number of patients presented mild and nonspecific abnormalities in a complete blood cell count and mild elevation of erythrocyte sedimentation rates and $\mathrm{C}$-protein reactive and liver enzyme levels. Serological evidence of acute rickettsiosis was observed in 19 patients $(61 \%)$. In $29 \%$ sera tested by polymerase chain reactions (PCRs) were positive. The sequence obtained from a PCR product revealed $98 \%$ identity with Rickettsia sp. strains RpA4, DnS14, and DnS28. All ticks removed from patients were PCR-positive. Sequencing showed 8 of them identified as $R$. slovaca and 2 as Rickettsia sp. strains RpA4, DnS14, and DnS28.
\end{abstract}

KEYWORDS: Rickettsia slovaca; Rickettsia sp.; strain RpA4; strain DnS14; DnS28 strain; tick; Dermacentor; DEBONEL; TIBOLA

\section{INTRODUCTION}

Ticks have been described as vectors of human infectious diseases since the beginning of the 20th century. In the last few years, different emerging tickborne diseases (TBDs) have been reported all over the world. The development

Address for correspondence: J. A. Oteo, Área de Enfermedades Infecciosas, Hospitales San MillánSan Pedro-de La Rioja, Avda. Viana No 1. 26001-Logroño (La Rioja), Spain. Voice: +34941297275; fax: +34941297267 .

e-mail: jaoteo@riojasalud.es

Ann. N.Y. Acad. Sci. 1078: 206-214 (2006). (C) 2006 New York Academy of Sciences. doi: 10.1196/annals.1374.040 
of new techniques, including the shell vial assay for isolation of organisms and molecular methods for their characterization, has allowed the etiology of many of these diseases to be known. ${ }^{1}$

Since 1990, we have investigated TBDs in the Hospital of La Rioja. Lyme borreliosis (LB) and Mediterranean spotted fever (MSF) are the most prevalent TBDs in our area, although some cases of human anaplasmosis have been described. $^{2}$

In November 1996 a 67-year-old man was evaluated for eschar on the thoracic region surrounded by an erythema (erythema migrans-like) after a tick bite. On examination he had several painful points of axillary lymphadenopathy. He kept the tick, which was identified as a female adult Dermacentor marginatus (D. marginatus). At this time, $D$. marginatus was only implicated as a vector of one case of LB. ${ }^{3}$ No antibodies against Borrelia burgdorferi, Rickettsia conorii, Anaplasma phagocytophilum, or Francisella tularensis were found. A skin biopsy was performed in order to investigate the presence of $B$. burgdorferi by polymerase chain reaction (PCR) and isolation in BSK medium, but without success. The PCR from the tick was also negative for B. burgdorferi. The patient was treated with doxycycline and signs and symptoms were soon alleviated. $R$. slovaca was identified by PCR from the frozen tick few years later. ${ }^{4}$ Since the description of this case, we revised all TBDs seen at our hospital up to that moment. In addition, we began to collect data and in same cases sera specimens from patients who presented with similar clinical findings.

\section{MATERIALS AND METHODS}

\section{Patients}

Patients' inclusion criteria were: (1) a crusted lesion or a point of necrosis (eschar) at the site of the tick attachment, surrounded by erythema and painful regional lymphadenopathy; and (2) tick bite by $D$. marginatus or a large tick during the period of maximum activity for $D$. marginatus (in our area from the end of October to the beginning of May).

The study was retrospective from January 1990 to December 2000 and prospective from January 2001 to December 2004.

Epidemiological and clinical data were obtained for patients studied prospectively. They were asked about the underlying diseases, date and site of the tick bite, method of removing the tick, geographical location and clinical features, evolution, and treatment. Routine laboratory tests (complete blood cell count, erythrocyte sedimentation rates, ASAT and ALAT, and C reactive protein) were performed. All these patients were reexamined 1, 4, 12, and 24 weeks after the initial visit. For the patients studied retrospectively these data were collected (when were available) from the clinical histories. 
Patients diagnosed with erythema migrans (LB) and MSF during 2003-2004 were used as controls.

\section{Ticks}

D. marginatus was collected from people: 10 from patients and 4 from asymptomatic persons.

\section{Microbiological Tests}

From 31 patients an acute-phase serum (before starting the treatment) and late-phase sera samples (1st, 3rd, and 6th months) were taken. From 21 patients an EDTA-blood sample was obtained. In eight cases skin biopsy specimens of the border of the lesion were also obtained. All samples were centrifuged and frozen at $-70^{\circ} \mathrm{C}$ until examination. All $D$. marginatus collected from people were classified with regard to genus and species and kept in $70 \%$ alcohol before being processed.

\section{Serological Tests}

Acute and convalescent sera samples were tested by an indirect immunofluorescence assay (IFA) for the presence of IgG antibodies against $R$. conorii (BioMérieux, Lyon, France) and Rickettsia slovaca (antigen slides kindly donated by Dr. Fátima Bacellar, Centro de Estudos de Vectores e Doenças Infecciosas do Instituto Nacional de Saúde, Portugal). Seroconversion, or a fourfold increase in the sera obtained in the late phase, was considered evidence of recent infection by spotted fever group (SFG) Rickettsia.

\section{Molecular Methods}

The acute-phase sera, the EDTA blood samples, the skin biopsy samples, as well as all $D$. marginatus specimens removed from people were analyzed by PCR assays.

DNA was extracted using the QIAamp Tissue kit (Qiagen, Hilden, Germany) according to the manufacturer' recommendations. The presence of SFG Rickettsia in human samples was determined by PCR assays for omp $A^{5}$ and $g l t A^{6}$ genes. All ticks were also screened for the presence of B. burgdorferi, A. phagocytophilum, and F. tularensis by the partial amplification of the 16S rDNA, $m s p 2$, and $17-\mathrm{kDa}$ lipoprotein genes, respectively. Sequencing reactions were carried out at Universidad de Alcalá de Henares (Spain). Sequences obtained were compared with those available at Genbank using BLAST utility (National Center for Biotechnology Information $<$ http://www.ncbi.nlm.nih.gov $>$ ). 


\section{RESULTS}

\section{Epidemiological and Clinical Data}

From January 1990 to December 2004, 54 patients who presented at our hospital with TBDs had the inclusion criteria for the study. The study was retrospective for 20 patients and prospective for 34 patients. Thirty-two patients $(59 \%)$ were female and $22(41 \%)$ were male. The median age was 37 years (range 3-78). All patients were bitten during the colder months (from the end of October to the beginning of May), with a peak in November (12 cases) and April (10 cases). No was observed from June to September. All patients remembered that they had been bitten by a "very large" tick. Fortyseven patients $(87 \%)$ removed the tick by hand, nearly always after previous administration of oil on the site of the tick attachment. Three of them kept the ticks. Seven patients were seen with the tick attached, which was removed by forceps. In all 10 cases the tick was identified as D. marginatus. All patients gave information about the geographical area where the tick had bitten them ( $96 \%$ in La Rioja Baja). The incubation period varied from 1 to 13 days (median 4.7). In 48 patients (90\%) the tick bite was located on the scalp, while 3 patients were bitten on the arms and 2 on the thorax. In all cases, the tick bite was located on the upper portions of the body. The typical skin lesion at the site of the tick bite started as a crusted lesion. A necrotic eschar ( 0.5 to $2.5 \mathrm{~cm}$ in diameter), surrounded by erythema, appeared a few days later. In eight cases $(15 \%)$ the erythema was higher than $5 \mathrm{~cm}$ (erythema migrans-like). In all patients whose tick bites were located on the scalp region, erythema was higher than $5 \mathrm{~cm}$ in diameter $(33 \mathrm{~cm}$ in one patient). Multiple and painful regional lymphadenopathy was the other major feature, being present in all cases. Only four patients presented with the skin lesion few days before the onset of lymphadenopathy. Most patients with the skin lesion on the scalp region suffered from headache. Low-grade fever $\left(<38^{\circ} \mathrm{C}\right)$ was observed in 18 patients $(33 \%)$, and 2 patients $(3.7 \%)$ had fever higher than $38.5^{\circ} \mathrm{C}$. A macular rash, which did not affect either palms or soles, was observed in only one patient. Routine laboratory tests were normal in most cases. Five of 47 patients $(10.6 \%)$ showed mild leukopenia and 2 of them $(4.2 \%)$ had increased white cell counts. The lowest and the highest leukocyte counts were $2,800 / \mathrm{mm}^{3}$ and $10,000 / \mathrm{mm}^{3}$ Three patients $(6.4 \%)$ suffered from thrombocytopenia. The lowest thrombocyte count was $100,000 / \mathrm{mm}^{3}$. One patient showed anemia ( $\mathrm{Hb}: 11 \mathrm{gr} / \mathrm{dL}$ ), and anemia and thrombocytopenia were observed in one case. Nine patients (19\%) had elevated erythrocyte sedimentation rates. C-reactiue protein was mildly elevated in $38 \%$ of cases (18 of 47). Eight of the 51 patients $(16 \%)$ had an elevated ASAT and ALAT level (less than twice the upper limit of the normal values). In all these cases the laboratory tests performed 1 month later gave normal results. 
All patients were treated with antibiotics. Doxycycline $(100 \mathrm{mg} / \mathrm{b} . \mathrm{i} . \mathrm{d} .14$ days) was administered to $79 \%$ of patients. Ten were treated with azithromycin $(10 \mathrm{mg} / \mathrm{kg}$ q.d. 5 days) and one child with josamycin $(500 \mathrm{mg} /$ b.i.d. 14 days). The treatment was changed in only one patient (doxycycline was substituted by azithromycin) on account of the development of nausea and diarrhea. In all cases learning of the signs and symptoms was observed. Evolution of disease was only available for 34 patients studied prospectively. Fever disappeared $48 \mathrm{~h}$ after the beginning of the treatment, and the painful lymphadenopathy remitted in 1 week for most patients. However, the evolution of the erythema and the eschar was slower, persisting both for 1 or 2 months. Sixty-six percent of patients fully recovered. The remaining ones developed persistent alopecia at the site of the tick bite $(0.5-2 \mathrm{~cm}$ in diameter $)$.

\section{Serological Tests}

IgG antibodies against $R$. conorii and $R$. slovaca were tested (IFA) in 31 patients. Evidence of recent infection by a Rickettsia SFG was observed in 19 patients $(61 \%)$. In 18 of them (95\%) seroconversion was detected. In one out of 19 patients $(5 \%)$ we observed a fourfold increase in IgG antibodies. In 27 cases $(87 \%)$ seroconversion or increase of the previous titers was detected during the first month. Four patients (13\%) showed seroconversion in the third month.

\section{PCR Assays}

All EDTA blood samples and skin biopsies were PCR-negative. In 9 of 31 sera tested (29\%) ompA PCRs were positive. Sequencing of PCR products was achieved in only one case. Comparison of the sequence with those deposited in Genbank revealed 98\% identity with a fragment of ompA gene of Rickettsia sp. strains RpA4, DnS14, and DnS28.

None of the $14 \mathrm{D}$. marginatus ticks removed from people was infected with A. phagocytophilum, B. burgdorferi, and F. tularensis. All of them were infected by SFG Rickettsia. By sequencing procedure (ompA gene) $R$. slovaca ( $100 \%$ similarity) was identified in 8 of 10 D. marginatus ticks removed from patients. Rickettsia sp. strains RpA4, DnS14, and DnS28 (98\% similarity) were identified in the two remaining ones, as well as in all D. marginatus removed from asymptomatic person.

\section{DISCUSSION}

This study describes the epidemiological, clinical, and laboratory findings for a new TBD in Spain. We first named the syndrome as Dermacentor-borne necrosis erythema lymphadenopathy (DEBONEL) because D. marginatus is 
the vector implicated in its transmission, and necrosis, erythema, and lymphadenopathy are the main clinical findings. ${ }^{4,7}$

All patients reported in this study were living in La Rioja, where LB and MSF are endemic. ${ }^{2}$ To estimate the specificity of epidemiological and clinical data of DEBONEL, we have also studied prospectively all patients diagnosed with erythema migrans (early located LB) and MSF at our hospital during 2003-2004. During this period, 27 cases of LB, 24 cases of DEBONEL, and 7 cases of MSF were diagnosed. Therefore, DEBONEL was a prevalent TBD in La Rioja during this period ( $21 \%$ of cases).

The seasonal distribution of DEBONEL was significantly different $(P<$ 0.05 ) from that in LB and MSF. DEBONEL occurred mainly during the colder months, whereas LB was diagnosed from late spring to early autumn, and MSF in summer. In addition, DEBONEL had a different geographical location. All cases were located in La Rioja Baja, in areas with low mountains (Wooded with Holm oaks) and with dry Indo-Mediterranean climate conditions.

In our study females were more frequently involved than males (female/male ratio: 1.45 ). On the contrary, LB and MSF were more frequent in males (female/male ratio: 0.5 and 0.16 , respectively) $(P<0.05)$. The patients' median age was 37 years. Nine patients $(16.6 \%)$ were children younger than 10 years. The average age of DEBONEL patients was significantly younger $(P<0.05)$ than the average age of LB (48 years) and MSF patients (55 years). In different Spanish studies, LB and MSF were also more frequent in males and their average age was higher. ${ }^{8-11}$ The site of the tick bite in DEBONEL was different from that LB and MSF: Patients diagnosed with DEBONEL were usually bitten on the scalp region $(90 \%)$, and always on the upper portions the body. However, the tick's attachment in LB and MSF can appear on any part on the body with predominance of the lower extremities ${ }^{12}(P$ $<0.05)$. These epidemiological differences between the three TBDs may be explained by the differences in the characteristics and behaviors of the tick vector.

Adult D. marginatus ticks' greatest activity, in La Rioja, is observed from early autumn to winter, ${ }^{13}$ which may explain why DEBONEL is more frequent during the colder months. The geographical distribution of the disease corresponds to the area of $D$. marginatus distribution in La Rioja. ${ }^{13}$ On the other hand, this species seems to prefer to live on hairy animals. To get a host, it waits higher than other genera (A. Estrada, personal communication). These facts could explain why children and females are more frequently involved than males, why the tick bite is usually located on the scalp region, and the fact that bites outside the head are observed only in males, on hairy regions (arms, axillas, and thorax).

The clinical symptoms of DEBONEL are different from those of LB and MSF. Nevertheless, some clinical features are similar in the three TBDs. Erythema migrans (the hallmark of the early located LB) later expands, central clearing occurs frequently, and it has a big size and does not have a central 
necrosis. However, erythema of DEBONEL is usually small (only $15 \%$ were higher than $5 \mathrm{~cm}$ ) and always has a central necrosis. General symptoms are rare and mild in both TBDs, except for headache, which is common in DEBONEL. Erythema and eschar at the inoculation site were significantly less common in patients with MSF than in patients with DEBONEL $(P<0.05)$. In addition, in MSF patients the eschar fully recovered and its size was smaller. On the contrary, some patients with DEBONEL, after healing of the eschar, developed a local alopecia. Regional lymphadenopathy was found less often in MSF than in DEBONEL $(P<0.05)$. The presence of fever and rash was also significantly less common in patients with DEBONEL than in patients with MSF $(P<0.05)$. MSF is usually a mild illness, but malignant forms have been reported in $5-12 \%$ of cases associated with $2.5 \%$ mortality. ${ }^{10,11}$ In this new TBD signs and symptoms were mild in all cases and we have not observed a fatal evolution in any case.

Similar epidemiological and clinical cases have been reported in France and Hungary by Raoult and Lakos. ${ }^{14-17}$ In Hungary the syndrome is known as tick-borne lymphadenopathy (TIBOLA).

In 1997 R. slovaca was identified by PCR in a skin biopsy from a French patient with similar clinical manifestations than those seen in La Rioja. ${ }^{14}$ In 2003 $R$. slovaca was isolated from a skin biopsy, which demonstrates its implications in the etiology of the disease. ${ }^{18}$

In the present study we have attempted to identify the causative agent of this new TBD in La Rioja. Culture was attempted from 2 skin biopsies, in serum and blood collected on heparin samples, and from a PCR-positive tick removed from a patient in other laboratories (Centro de Estudos de Vectores e Doenças Infecciosas do Instituto Nacional de Saúde, Portugal and Unité des Rickettsies, Marsella, France) without success. Nevertheless, we have confirmed the SFG rickettsial infection by PCR in $29 \%$ of sera tested, and in all ticks (10) removed from patients. By means of a sequencing procedure $R$. slovaca $(100 \%$ similarity) was identified in 8 of $10 \mathrm{D}$. marginatus ticks removed from patients. Rickettsia sp. strains RpA4, DnS14, and DnS28 (98\% similarity) were identified in the two remaining ones, as well as in a serum sample from a patient.

Rickettsia sp. strains RpA4, DnS14, and DnS28 are closely related and branched with members of the $R$. massiliae group. These strains were first identified in Dermacentor sp. ticks collected in territories of the former Soviet Union. ${ }^{19,20}$ The prevalence of Rickettsia sp. strains RpA4, DnS14, and DnS28 in D. marginatus collected from people and from several animal hosts in La Rioja is high (59.3\%). Six of $14(43 \%)$ D. marginatus ticks removed from people were infected by these strains. Moreover only 2 of 6 people (33\%) developed disease. The remaining ones (4/6) did not develop any clinical manifestation.

The pathogenicity of Rickettsia sp. strains RpA4, DnS14, and DnS28 is still unknown and, up to date, these genotypes have not been implicated in human disease. 
Our data suggest that $R$. slovaca is the main etiological agent of DEBONEL in La Rioja. Nevertheless, it is possible that other Rickettsia sp., such as strains RpA4, DnS14, and DnS28 may also be implicated in the etiology.

On the other hand, according to our results, DEBONEL may be a prevalent TBD in Spain and should be suspected following a tick bite. In addition, persons bitten by $D$. marginatus infected with $R$. slovaca had a higher risk of developing DEBONEL than did those bitten by D. marginatus infected with Rickettsia sp. strains RpA4, DnS14, and DnS28 (100\% and 33\%, respectively).

\section{ACKNOWLEDGMENTS}

This study was supported in part by grants from the Fondo de Investigación Sanitaria (FIS PI021810 and FIS G03/057), Ministerio de Sanidad y Consumo, Spain.

\section{REFERENCES}

1. Parola, P. \& D. Raoult. 2001. Ticks and tick-borne bacterial diseases in humans: an emerging infectious threat. Clin. Infect. Dis. 32: 897-928.

2. Отео, J.A. 2001. Tick-borne diseases in Spain. Clin. Microbiol. Infect. 7: 31.

3. Angelov, L., P. Dimova \& W. Berbencova. 1996. Clinical and laboratory evidence of the importance of the tick D. marginatus as a vector of $B$. burgdorferi in some areas of sporadic Lyme disease in Bulgaria. Eur. J. Epidemiol. 12: 449-506.

4. OteO, J.A. \& V. IBARRA. 2002. DEBONEL (Dermacentor-borne-necrosiserythema-lymphadenopathy): una nueva enfermedad transmitida por garrapatas? Enferm. Infecc. Microbiol. Clin. 2: 51-52.

5. Fournier, P.E., V. Roux \& D. Raoult. 1998. Phylogenetic analysis of spotted fever group rickettsiae by study of the outer surface protein rOmpA. Int. J. Syst. Bacteriol. 48: 839-849.

6. Roux, V., E. Rydkina, M. EREMEeva, et al. 1997. Citrate synthase gene comparison, a new tool for phylogenetic analysis, and its application for the Rickettsiae. Int. J. Syst. Bacteriol. 47: 252-261.

7. Oteo, J.A., V. Ibarra, J.R. Blanco, et al. 2004. Dermacentor-borne necrosis erythema and lymphadenopathy: clinical and epidemiological features of a new tick-borne disease. Clin. Microbiol. Infect. 4: 327-331.

8. Oteo, J.A. \& V. Martínez de Artola. 1995. Borreliosis de Lyme: aspectos epidemiológicos y etiopatogénicos. Enferm. Infecc. Microbiol. Clin. 13: 550-555.

9. Oteo, J.A., J.R. BlanCo, V. Martínez de Artola, et al. 2000. Eritema migratori (borreliosis de Lyme): características clínico epidemiológicas de 50 casos. Rev. Clin. Esp. 2: 57-59.

10. Jufresa, J., J. Alegre, J.M. Surinach, et al. 1997. Clinical study of 86 patients with Mediterranean boutonneuse fever who were admitted in a university general hospital [in Spanish]. An. Med. Int. 14: 328-331.

11. Antón, E., B. Font, I. SANFEliú, et al. 2003. Clinical and laboratory characteristics of 144 patients with Mediterranean Spotted Fever. Eur. J. Clin. Microbiol. Infect. Dis. 22: $126-128$. 
12. Oteo, J.A., V. Ibarra, J.R. Blanco, et al. 2003. Epidemiological and clinical differences among DEBONEL/TIBOLA and other tick-borne diseases in Spain. Ann. N. Y. Acad. Sci. 990: 391-392.

13. Oteo, J.A., J.R. Blanco, R. Grandival, et al. 1998. Ixodidiasis humana en La Rioja: estudio estacional y de especies. Presented at the I Reunión Nacional del Grupo de Rickettsias y Borrelias. Haro, La Rioja, Spain.

14. Raoult, D., P.H. Berbis, V. Roux, et al. 1997. A new tick-transmitted disease due to Rickettsia slovaca. Lancet 350: 112-113.

15. LAKOS, A. 1997. Tick-borne lymphadenopathy-a new rickettsial disease? Lancet 350: 1006.

16. Raoult, D., A. Lakos, F. Fenollar, et al. 2002. A spotless rickettsiosis caused by Rickettsia slovaca and associated with Dermacentor ticks. Clin. Infect. Dis. 34: 1331-1336.

17. LaKOS, A. 2002. Tick-borne lymphadenopathy (TIBOLA). Wien. Klin. Wochenschr. 114: 648-654.

18. Cazorla, C., M. Enea, F. Lucht, et al. 2003. First isolation of Rickettsia slovaca from a patient, France. Emerg. Infect. Dis. 9: 135.

19. Rydkina, E., V. Roux, N. Fetisova, et al. 1999. New Rickettsiae in ticks collected in territories of the former Soviet Union. Emerg. Infect. Dis. 5: 811-814.

20. Sphynov, S., P. PARola, N. Rukadov, et al. 2001. Detection and identification of spotted fever group Rickettsiae in Dermacentor ticks from Russia and Central Kazakhstan. Eur. J. Clin. Microbiol. Infect. Dis. 20: 903-905.

21. Oteo, J.A., A. Portillo, S. Santibáñez, et al. 2006. Prevalence of spotted fever group Rickettsia (SFGR) species detected in ticks in La Rioja, Spain. Ann. N. Y. Acad. Sci. this volume. 